\title{
A SURVEY OF CLOUD SUPPORTER: A LOAD BALANCING FRAMEWORK IN CLOUD USING SMARTPHONE
}

\author{
Anantkumar Vikas Salame ${ }^{1}$, Prof. Pradnya V. Kulkarni ${ }^{2}$ and Prof. Shakti S. Kinger ${ }^{3}$
}

Abstract- The main objective of this paper was to propose cloud supporter framework to support cloud for
processing multiple tasks. Because of multiple tasks on cloud server, it works slower and sometimes gets
failed. In this situation distributing or scheduling of tasks on the distributed computing system is the only
solution to reduce workload on server. Computing capability of smart phone's CPU can compete with the CPU
of the computers. Smart phones are energy-efficient and cost-effective alternative to running certain tasks of
traditional servers. Distributing tasks for computations must utilize all the resources equally, no resource should
be under or over utilize this problem leads to focus on the load balancing technique to support the cloud for
processing tasks. The approach of this paper was surveying about the load balancing frame work as cloud
supporter for processing tasks on smart phones when they are plugged into the power sources.
Keywords- Cloud supporter, distributed computing, load balancing, CWC, android volunteer

\section{INTRODUCTION}

Every enterprise in the world has the basic need of fast server to work efficiently as well as rapidly. But day by day computational workloads on server in the form of multiple task requests are also increasing. Because of these multiple tasks on cloud server, it works slower and sometimes gets failed. In this present situation distributing or scheduling of tasks on the distributed computing system is the only solution to reduce workload on server.

To form the best distributed computing system we are using smart phones as volunteer or android clients, there are many other options to form distributed system such as vehicular cloud [5] or virtual machines [8] but computing capability of smart phone's CPU can compete with the CPU of the computers [6]. Smart phones are energy-efficient and cost-effective alternative to running certain tasks of traditional servers. When these smart phones are plugged into power source for charging battery then this idle phones provides the increasing computing capabilities and sizable computing infrastructure. So we are using CWC (computing while charging) architecture for distributed computing infrastructure.

Distributing tasks for computations must utilize all the resources equally, no resource should be under or over utilize this problem leads to focus on the load balancing technique to support the cloud for processing tasks. In this paper we are proposing the load balancing frame work as cloud supporter for processing tasks on smart phones when they are plugged into the power sources.

We name our framework Cloud supporter, this framework is supporting cloud server to handle multiple task request more efficiently by distributing tasks to its connected volunteer. Volunteers

${ }^{1}$ Dept. of Computer Engineering, Maharashtra Institute of Technology, Pune

${ }^{2}$ Dept. of Computer Engineering, Maharashtra Institute of Technology, Pune

${ }^{3}$ Dept. of Computer Engineering, Maharashtra Institute of Technology, Pune 
are the android smart phone which will accept the task and process it, after completing the task processing result will get back to the cloud server and server will send this result to the task submitter.

About CWC architecture, CWC stands for computing while charging as the biggest problem of using smart phones for computing are battery life and bandwidth. If these smart phones are using for the heavy computation during in use by its owner then battery of phone may drains very fast and it will be unusable after some time. To overcome this problem the solution is using smart phones when they are being charged at night, when active use by phone owners is not likely. CWC uses a single server connected to the Internet, for scheduling jobs on the smart phones and collecting the outputs from the computations [1].

\section{RELATED WORK}

While use of smart phones for computing has been proposed many times in various contexts, there are very few studies that share our vision of using the computing power of smart phones for executing enterprise-grade computations. In that follows, we compare the similarities and differences between our work and these previous proposals.

The system that is nearly similar to our work is CANDIS; In CANDIS framework authors proposed use of employee smart phones for executing enterprise applications [2]. They enforced an execution setting for automaton that permits for running desktop Java applications on smart phones in an automatic fashion [1]. Also created similar observations about scheduling tasks based on processing capabilities of smart phones. While we envision similar applications and system implementation, we provide an algorithm that helps to minimize the completion time of the job based on both CPU capabilities and bandwidths of smart phones, which has not been addressed in CANDIS.

The well-known problem of scheduling jobs on a set of processors in a cluster for the makespan objective or we can say the completion time of the last job, this drawback has been shown to be NP-Hard and several heuristics have already been planned to reduce the execution time. For that we introduce an approach based on linear programming algorithm, The idea is to relax an integer number linear program and use $\ell \mathrm{p}$ norm-based [3] operators to force the solver to search out almost-integer solutions which will be assimilated to number solution. We have a tendency to think about the case wherever jobs are either rigid or moldable. A rigid parallel job is performed with a predefined number of processors while a moldable job will outline the quantity of processors that it is using just before it starts its execution. We tend to compare the scheduling approach with the classic Largest Task 1st list based algorithm and we will show that our approach provides sensible results for little instances of the problem.

Distributed load balancing is a very important system operates destined to distribute workload among accessible processors to boost throughput and/or execution times of parallel pc in Cluster Computing [4]. Rather than balancing the load in cluster by process migration or by moving a whole process to a less loaded pc, we tend to build an effort to balance load by making partition of processes into separate jobs then balance them to android volunteers of cluster and to form cluster fuzzy c-means (FCM) algorithm is the novel approach than the clustering algorithm such as k-means algorithm [4].

Advantages- By considering the related work, our approach helps to minimize the completion time of the job based on both CPU capabilities and bandwidths of smart phones which has not been addressed in previously. Also we are tapping the complete use of computation capability of smart phones which has sizable computing infrastructure when they are in idle mode and plugged into the charging socket with the help of CWC architecture. 
When these smart phones can be use for processing the task of cloud server of various enterprises then it will prevent the server from getting slow or failed by reducing the workload on the server also it becomes the cost cutting factor when comparing to cost of uptime server.

The load balancing framework will work as cloud supporter to ensure that all the available resources in the distributed computing system should be utilize equally.

\section{EXISTING AND PROPOSED SYSTEM}

Talking about the existing system related to our work the system that is nearly similar is CANDIS; In this framework authors proposed use of employee smart phones for executing enterprise applications [2]. They enforced an execution setting for automaton that permits for running desktop Java applications on smart phones in an automatic fashion also created similar observations about scheduling tasks based on processing capabilities of smart phones [1].

Also in the CWC framework they used the increasing computing capability of the smart phones by plugging it into the power source which is also similar in our work but in addition we are using clustering technique to form the cluster of android volunteer having different processing capabilities on account of their capabilities load will be distributed to the volunteer so that they can also provide good performance while processing the given task.

In our proposed architecture of cloud supporter framework there will be a submitter who will submit the task to the cloud server for processing. Before submitting the task submitter has to register itself to the cloud server with proper credential details. After logging in submitter can submit the different tasks such image processing or file encryption/decryption etc.

We are using the image processing task for easily understanding the concept and efficiency of our work. Cloud server will accept the task and distribute it to the available volunteers for processing. If there is unavailability of volunteers then server will send the toast massage for plugging smart phone on charge and being ready to process the task on registered android volunteer as because we are using the CWC architecture. Smart phones needs to submit their parameters to work as volunteer and all the log details of volunteers are separately saved in the database connected to the cloud server.

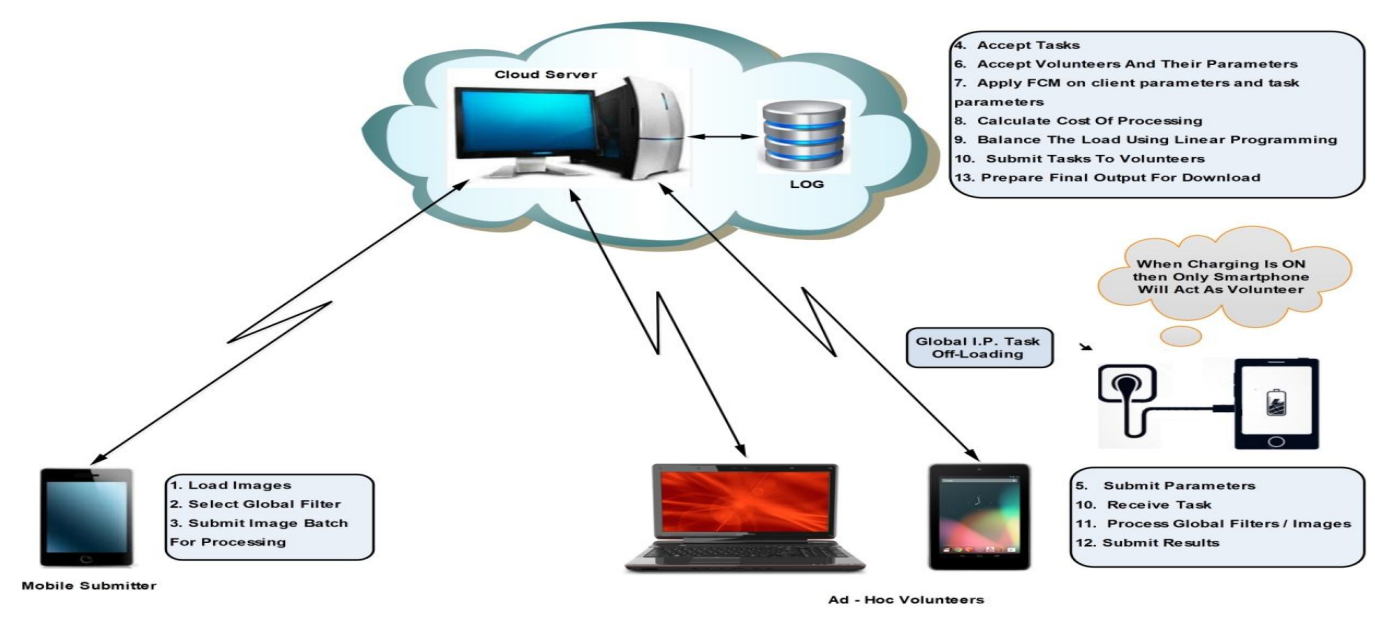

Fig. - Cloud supporter architecture

Cloud server will accept the parameters of task and clients and apply the FCM algorithm to form the cluster of volunteers according to their computing capabilities. Only to show the different 
computing capabilities of volunteer we are using a laptop as volunteer. By calculating the cost of processing load will be balance using the linear programming algorithm and rigid and moldable tasks will schedule to the volunteers. Volunteers process these tasks completely and result is send back to the cloud server, finally server will prepare the final output to download by the submitter of the task.

\section{CONCLUSION}

According to the survey related to this work, it is found that distributed computing system is the best option to handle the multiple task requests on cloud server. Also to support cloud server for processing these tasks the best and novel idea is using the smart phones as they has good computing capability that can compete with capability of computer. Use of CWC architecture provides the increasing processing capabilities of smart phone and sizable computing infrastructure. The load balancing framework is necessary to completely utilize all the available resources and equal distribution of the load according to the computing capabilities of the resources to ensure that there should not be any under or over utilized resource. So this load balancing framework works as a cloud supporter to reduce its workload.

\section{REFERENCES}

[1] M.Y. Arslan, I. Singh, S. Singh, H.V. Madhyastha, K. Sundaresan and S.V. Krishnamurthy "CWC: A Distributed Computing Infrastructure Using Smartphones" In Proc. IEEE Transaction on mobile computing, vol. 14, no. 8, august 2015

[2] S. Schildt, F. Büsching, E. Jörns, and L Wolf "CANDIS: Heterogenous Mobile Cloud Framework and Energy Cost-Aware Scheduling" In Proc. IEEE International Conference on Green Computing and Communications and IEEE Internet of Things and IEEE Cyber, Physical and Social Computing 2013

[3] S. Chretien1, J. Nicod, L. Philippe and V. Rehn-Sonigo "Job Scheduling Using successive Linear Programming Approximations of a Sparse Model” In Proc. EuroPar 2012. 2012.feb 2013

[4] N. Nehra, R.B. Patel, V.K. Bhat "A Framework for Distributed Dynamic Load Balancing in Heterogeneous Cluster” In Proc. Journal of Computer Science 3 (1): 14-24, 2007

[5] H. Zhang, Q. Zhang, and X. Du "Toward Vehicle-Assisted Cloud Computing for Smartphones" In Proc. IEEE Transactions on Vehicular Technology 2015

[6] S. Harizopoulos and S. Papadimitriou "A Case for Micro-Cellstores: Energy-Efficient Data Management on Recycled Smartphones" In 2011

[7] S. Ray and A. De Sarkar "Execution Analysis Of Load Balancing Algorithms In Cloud Computing Environment" In Proc. International Journal on Cloud Computing: Services and Architecture (IJCCSA), Vol.2, No.5, October 2012

[8] S. G.Damanal and G. R. M. Reddy "Optimal Load Balancing in Cloud Computing By Efficient Utilization of Virtual Machines” In IEEE 978-1-4799-3635-9/14 2014

[9] S. Sethi, A. Sahu, and S. K. Jena "Efficient load Balancing in Cloud Computing using Fuzzy Logic" In Proc. IOSR Journal of Engineering (IOSRJEN) ISSN: 2250-3021 Volume 2, Issue 7(July 2012), PP 65-71

[10] M. Katyal and A. Mishra "A Comparative Study of Load Balancing Algorithms in Cloud Computing Environment" In Proc. International Journal of Distributed and Cloud Computing Volume 1 Issue 2 December 2013

[11] K.Al Nuaimi, N. Mohamed, M. Al Nuaimi and J. Al-Jaroodi "A Survey of Load Balancing in Cloud Computing: Challenges and Algorithms"

[12] In Proc. IEEE Second Symposium on Network Cloud Computing and Applications 2012

[13] N. Rajovic, P. M. Carpenter, I. Gelado, N. Puzovic, A. Ramirez, and M. Valero "Supercomputing with Commodity CPUs: Are Mobile SoCs Ready for HPC?” November 2013 